\title{
Competition Reduces Response Times in Multiparty Conversation
}

\author{
Judith Holler ${ }^{1,2 *}$, Phillip M. Alday ${ }^{1}$, Caitlin Decuyper ${ }^{1}$, Mareike Geiger $^{1,2}$, \\ Kobin H. Kendrick ${ }^{3}$ and Antje S. Meyer ${ }^{1,2}$ \\ ${ }^{1}$ Max Planck Institute for Psycholinguistics, Nijmegen, Netherlands, ${ }^{2}$ Donders Institute for Brain, Cognition, and Behavior, \\ Radboud University, Nijmegen, Netherlands, ${ }^{3}$ University of York, York, United Kingdom
}

\section{OPEN ACCESS}

Edited by:

Marianne Gullberg,

Lund University, Sweden

Reviewed by:

Herbert H. Clark

Stanford University, United States

Mattias Heldner,

Stockholm University, Sweden

*Correspondence:

Judith Holler

Judith.holler@mpi.n

Specialty section:

This article was submitted to

Language Sciences,

a section of the journal

Frontiers in Psychology

Received: 09 April 2021

Accepted: 04 August 2021

Published: 16 September 2021

Citation:

Holler J, Alday PM, Decuyper C, Geiger $M$, Kendrick KH and Meyer AS

(2021) Competition Reduces

Response Times in Multiparty

Conversation

Front. Psychol. 12:693124.

doi: 10.3389/fpsyg.2021.693124
Natural conversations are characterized by short transition times between turns. This holds in particular for multi-party conversations. The short turn transitions in everyday conversations contrast sharply with the much longer speech onset latencies observed in laboratory studies where speakers respond to spoken utterances. There are many factors that facilitate speech production in conversational compared to laboratory settings. Here we highlight one of them, the impact of competition for turns. In multiparty conversations, speakers often compete for turns. In quantitative corpus analyses of multi-party conversation, the fastest response determines the recorded turn transition time. In contrast, in dyadic conversations such competition for turns is much less likely to arise, and in laboratory experiments with individual participants it does not arise at all. Therefore, all responses tend to be recorded. Thus, competition for turns may reduce the recorded mean turn transition times in multi-party conversations for a simple statistical reason: slow responses are not included in the means. We report two studies illustrating this point. We first report the results of simulations showing how much the response times in a laboratory experiment would be reduced if, for each trial, instead of recording all responses, only the fastest responses of several participants responding independently on the trial were recorded. We then present results from a quantitative corpus analysis comparing turn transition times in dyadic and triadic conversations. There was no significant group size effect in question-response transition times, where the present speaker often selects the next one, thus reducing competition between speakers. But, as predicted, triads showed shorter turn transition times than dyads for the remaining turn transitions, where competition for the floor was more likely to arise. Together, these data show that turn transition times in conversation should be interpreted in the context of group size, turn transition type, and social setting.

Keywords: multi-party conversation, turn-taking, turn transitions, competition, response times, response latencies

\section{INTRODUCTION}

In everyday conversation, speakers' turns are well coordinated in time. As noted in a seminal article by Sacks et al. (1974), turns alternate such that most of the time only one person talks, and the gaps and overlaps between their turns are short. For instance, for a corpus of polar (yes/no) questions in ten languages Stivers et al. (2009) found a mean turn transition duration of around 200 ms when averaging across languages and little variation between languages. For a corpus of Dutch, English, and Swedish conversations Heldner and Edlund (2010) reported similar values. An important 
issue in current psycholinguistic work on conversation is how interlocutors achieve this tight coordination of their turns. The short turn transition times are remarkable because they contrast sharply with the much longer speech onset latencies observed when participants in psycholinguistic experiments produce simple utterances. For instance, naming an object takes at least $600 \mathrm{~ms}$ (e.g., Indefrey and Levelt, 2004) and planning a sentence describing a scene can easily take more than a second (Griffin and Bock, 2000; Konopka, 2012). How can speakers be so fast to respond to each other in conversation when it takes them so long to plan simple utterances in the laboratory? As we review below, many variables have already been identified that likely support fast turn-taking in natural conversation. In the present paper, we explore the impact of a variable that has not received much attention in the literature: competition for turns, which, we argue, shortens turn transition times in multi-party conversations compared to dyadic conversations. It may also contribute to explaining the discrepancy between long response times in laboratory studies of speech planning and short turn transition times in corpora of conversational speech.

Levinson and Torreira (2015; see also Levinson, 2016) proposed that speakers achieve fast transitions between their turns by being proactive: They do not await the end of the partner's turn, but begin to plan a response as soon as possible. For example, a guest in a restaurant hearing the waiter say "Can I.?" may often be able to guess the continuation of the question (“...get you anything else?”) or at least its gist, and plan a response after the first couple of words. This Early Planning Hypothesis plays a central role in Levinson and Torreira's model of turn taking. Interlocutors begin to plan a response to their partner as soon as they have sufficient information to do so and launch it when the end of the turn is imminent.

Several laboratory studies have examined whether speakers indeed already begin to plan an utterance while still listening to their partner. This is not self-evident because both comprehending and planning utterances require cognitive resources, and because the conceptual and linguistic encoding processes may interfere with each other (e.g., Ferreira and Pashler, 2002; Cleland et al., 2012; Boiteau et al., 2014). In these studies, participants heard utterances and had to respond as quickly as possible. The critical manipulation was that the cue to the answer appeared either early or late in the utterance. If, as the Early Planning Hypothesis predicts, utterance planning begins as soon as possible, the response times should be shorter when the cue appears early than when it appears late in the utterance. As an example, in the first study of this type, Bögels et al. (2015) used quiz questions, where the cue to the answer appeared early, as in "Which character, also called 007, appeared in the famous movies?" or late, as in "Which character from the famous movies is also called 007?" They found that the participants responded faster, on average by about $300 \mathrm{~ms}$, in the former than in the latter condition (means: 640 versus $950 \mathrm{~ms}$ ). This suggests that they must have begun planning their utterances earlier in the early than in the late cue condition, as predicted by Levinson and Torreira's Early Planning Hypothesis. Later studies using similar paradigms provided further strong support for this hypothesis
(Barthel et al., 2016; Bögels et al., 2018; Corps et al., 2018, 2019; Meyer et al., 2018).

Though these studies unambiguously supported the Early Planning Hypothesis, they consistently failed to elicit latencies that were as short as the mean turn transitions obtained in quantitative analyses of conversational corpora. These are, as noted above, often around $200 \mathrm{~ms}$. For instance, in the study by Bögels et al. (2015), the mean response time in the early cue condition was $640 \mathrm{~ms}$; in the study by Barthel et al. (2016) it was $806 \mathrm{~ms}$; and in the study by Corps et al. (2018, Experiment 2b) it was $484 \mathrm{~ms}$. This discrepancy indicates that early planning by itself does not suffice to explain the speed of conversational turntaking and that there must be other factors at play that are absent in typical laboratory experiments.

A number of potentially important factors have been discussed in the literature. For instance, in natural conversation speakers can often refer to mutually shared knowledge (also called "common ground"), which facilitates understanding and the generation of appropriate responses (Clark, 1996; BrownSchmidt et al., 2015). Relatedly, interlocutors may converge in their use of words and phrases. Over the course of an interaction, this may lead to increasing alignment of their conceptual and linguistic representations, which facilitates mutual understanding and responding (e.g., Pickering and Garrod, 2004; Garrod and Pickering, 2009; Branigan and Pickering, 2017; but see Healey et al., 2014). Such alignment can also arise in laboratory contexts (e.g., Garrod and Anderson, 1987; Brennan and Clark, 1996; Branigan et al., 2000). However, often the semantic and/or syntactic structure of the utterances to be produced in a laboratory study is largely predetermined, preventing spontaneous facilitatory alignment (e.g., Sjerps and Meyer, 2015; Barthel et al., 2017). Furthermore, conversation often features very short contributions ("yeah") and utterances beginning with turn-initial particles, which are likely to be fast to initiate, but do not appear in typical laboratory speech (e.g., Knudsen et al., 2020). Finally, natural conversations are often multimodal (e.g., including manual, head and facial gestures in addition to speech) and the presence of visual information may substantially facilitate the comprehension and production of utterances (Holler et al., 2018). In contrast, most psycholinguistic experiments assessing the Early Planning Hypothesis have allowed the use of spoken language only.

In short, there are many potential reasons why laboratoryrecorded response times are much longer than turn transition times in conversation. In this paper, we draw attention to a factor that seems to have been largely overlooked so far: competition for turns, which may arise in multi-speaker conversations but is much less likely to occur in dyadic conversations and is absent from standard laboratory experiments where participants are tested individually.

To elaborate, in a standard psycholinguistic experiment, participants are tested individually and all of their responses (perhaps with the exclusion of errors and some outliers, i.e., responses with extremely short or long latencies) are recorded and averaged. The same is likely to apply in analyses of dyadic conversations. Here participants abide by common turn-taking rules, which involves that the current speaker gives the other 
speaker(s) the opportunity to take the turn before continuing to speak themselves (Sacks et al., 1974). This means that in quantitative corpus analyses of dyadic conversations all speakers' planned responses (except perhaps for some responses with very pronounced delays foreshadowing dispreferred responses; see Kendrick and Holler, 2017) tend to be realized and recorded.

The situation is different in multi-party conversations. Here several speakers may wish to speak but typically only one person, the fastest responder, obtains the next turn (Sacks et al., 1974). Thus, those turns that may have been planned but were never issued because someone else responded faster will not enter into the analysis. Alternatively, two or more speakers may start to speak almost at the same time, but in a quantitative corpus analysis the turn transition time would typically still be the fastest responder's time, or such cases would be removed from the analysis altogether. In short, in multi-party conversation, only the fastest of the competing responses are included in the mean turn transition time, whereas the mean for dyadic conversations is based on almost all responses.

To illustrate the impact of this sampling bias on typical response times we describe the results of simulations showing how the mean and median response times from a laboratory experiment would change, relative to the mean and median of all response times, if for each trial only the fastest response times from two or more participants were recorded and averaged. For instance, if participant $A$ had a response time of $650 \mathrm{~ms}$ and participant B had a response time of $700 \mathrm{~ms}$, either both latencies or only A's latency would be entered into the dataset. These simulations show the impact of recording all or a subset of the response times and combining them in a mean or median. They do not concern the ways the speakers' behavior may change when they talk in smaller or larger groups.

Then we turn to actual conversations. As discussed above, in quantitative analyses of multi-party conversation, the recorded turn transition time is the response time of the fastest speaker. It follows that as more people participate in a conversation, the mean turn transition time, which is the mean fastest response time, should decrease even when the individual speakers' behavior is not affected by the number of participants. The same holds for the median. Of course, the speakers' behavior may change as well. For instance, speakers may begin to plan or launch utterances earlier as they compete for the floor with more co-participants. Although conversation as a whole may be characterized as collaborative, there is often a competitive element to turn-taking (French and Local, 1983; Schegloff, 2000; Kurtić et al., 2013), arising perhaps most clearly in multi-party conversation (e.g., Sacks et al., 1974, p. 712).

No systematic quantitative comparison of turn transitions in dyadic and multi-party conversations appears to exist to date. The existing quantitative studies of turn transition times focused on dyads (e.g., ten Bosch et al., 2004; Heldner and Edlund, 2010; Roberts et al., 2015) or on multi-party conversations (Girard-Groeber, 2015; Holler et al., 2018) or did not distinguish between dyadic and multi-party conversations in their analyses (e.g., Stivers et al., 2009; de Vos et al., 2015). The effect of competition for the floor on turn transition times has been investigated by comparing dyads interacting in friendly chats or arguments, with the latter resulting in shorter turn transitions (Trimboli and Walker, 1984), but not in multi-party compared to dyadic conversation.

To begin to fill this gap in the literature, we present an analysis of a corpus of unscripted casual conversations investigating the effect of group size (dyadic versus triadic) on turn transition times. The conversations were recorded in a laboratory, and the participants in the dyadic conversations also participated in the triadic conversations. We distinguished between two types of turn transitions, question-response sequences (QR transitions hereafter) and non-question-response turn transitions (non-QR transitions). The rationale for this is that questions often specify a specific respondent even in multi-party settings (e.g., Holler and Kendrick, 2015) or address several participants as one collective unit (Lerner, 1993). This may reduce the competitive element of turn-taking compared to non-QR transitions. In nonQR transitions, the current speaker may also select the next speaker, but speakers may more frequently self-select. Hence competitive effects should be seen more clearly in non-QR than in QR transitions. Note that in the present study we do not distinguish between competitive overlap (i.e., overlap that would be perceived as interruptive) and non-competitive overlap (Ferguson, 1977; Beattie, 1982; French and Local, 1983; Schegloff, 2000, 2001). This distinction is undoubtedly an important one for understanding the phenomenon of overlap and its management in interaction. However, the focus of the present study is on turn transition times, that is, a continuous measure of the extent of the gap or overlap that occurs between speakers. This measure is related to previous studies that have measured response times in conversational corpora (see above) which also did not consider different types of overlap. For comparability, we here apply the same general measure.

In sum, we propose that turn transition times in multiparty conversation may be reduced for two related reasons: First, there is a statistical reason; the fastest of several planned responses determines the recorded turn transition time. Second, the speakers' behavior may change when they compete for the turn. Note that we are not proposing that competition for the floor is the only, or even the most important, cause of short turn transition times in multi-party conversation. We view it as one factor that may be influential but has so far received little attention in the psycholinguistic literature on conversation.

\section{STUDY 1: SIMULATIONS}

The aim of the simulations was to examine how much the response time distribution obtained in a laboratory experiment would change if, instead of recording all responses, for each trial only the fastest response from two or more participants were recorded. The observed response times, on which the simulations were based, come from an experiment by Meyer et al. (2018, Experiment 1), which we briefly describe below.

\section{Experiment and Dataset}

The participants were 21 adult native speakers of Dutch. The data from one participant were excluded due to technical failure. The 
participants were asked to listen to recorded questions about the objects on their screen (e.g., "Heb je een groene sweater?" English "Do you have a green sweater?") and to respond as quickly as possible with "ja” (yes) or "nee" (no). There were always four objects on the screen. The referent object (the sweater in the example) was always included in this four-object display, but not necessarily in the color mentioned in the question. There were two experimental conditions, called the monochrome and the multi-color condition. In the monochrome condition all objects had the same color. In the multi-color condition they had different colors. This affected when the participants could begin to plan their response. For instance, when the question was about a green sweater and all objects on the screen were green, the participants could prepare to answer affirmatively as soon as they had comprehended the color adjective. Similarly, when all objects were white, the participants could begin to prepare a negative answer as soon as they had comprehended the adjective. In contrast, when the four objects on the screen had different colors, the participants could only begin to prepare a response after they had comprehended the noun as well. Thus, the Early Planning Hypothesis predicts that the response times should to be shorter in the monochrome than in the multicolor condition. The experiment included 256 trials, with 128 monochrome and 128 multi-colored displays. 64 monochrome and 64 multi-colored displays required an affirmative answer. The remaining displays required a negative answer.

A native Dutch speaker transcribed the utterances. Incorrect responses (less than $3 \%$ of the responses) and outliers, i.e., responses times deviating by more than 2.5 standard deviations (SD) from the condition mean $(0-3 \%$ of the responses per condition) were excluded from the analyses. Table 1 shows the results for the remaining responses. As expected, participants were faster to respond in the monochrome condition than in the multi-color condition. In addition, affirmative answers were overall given faster than negative ones. The benefit for affirmative over negative answers was smaller in the monochrome than in the multi-color condition. For the statistical analysis and a discussion of the findings, please refer to the original paper.

\section{Methods}

For the simulations, we created simulated pairs of participants, and for each simulated trial selected the fastest response time: First, we paired each of the 20 participants with each of the other participants, yielding 190 pairs. To obtain simulated data for each trial, response times were selected at random from each participant's data per condition (for instance from the response times in the monochrome affirmative condition). Thus for each trial, two response times, one from each participant, were selected. This was done 32 times per condition, simulating the 32 trials of the experiment. For the entire experiment, this yielded a dataset of 44,442 response time pairs. The shorter of the response times was selected and plotted as the "winning" response time per trial.

\section{Results and Discussion}

Figure 1 shows as density plots how the observed response times per condition (in blue) and the simulated "winning" response times (in orange) were distributed. As can be seen, the distributions of "winning" response times peaked earlier and had thinner right tails, i.e., included fewer long response times.

The simulated condition means and medians of the winning response times are tabulated in Table 1 next to the empirically observed means and medians for all responses. The two rightmost columns of the table show the gain, that is the differences between the means and median observed in the experiment and in the simulation.

As the numbers in the gain columns show, in the multi-color conditions, the estimated mean response time was shorter by $92 \mathrm{~ms}$ in both the affirmative and negative response condition for the "winning" responses compared to all responses. The medians changed less, by 28 and $48 \mathrm{~ms}$ for affirmative and negative responses, respectively. This is because medians are less affected by extreme values than means. In the comparison of the two response times per trial and the selection of the fastest one the longest response times are most likely to be discarded.

In the monochrome conditions, the response distributions were bimodal, as can be seen in the density plots. This is because here participants could, but did not have to, respond before the end of the question. As all objects on the screen had the same color, they could respond as soon as they had understood the adjective ("Do you have a green?"), which yielded response onsets shortly before the end of the question (“.sweater?"). However, participants often only responded after they had heard the entire question, yielding much later response onsets. The average reduction in mean response time was $149 \mathrm{~ms}$ for affirmative and $128 \mathrm{~ms}$ for negative responses. The medians were reduced by 66 and $75 \mathrm{~ms}$, respectively.

In further simulations we assessed how the typical "winning" response times changed when responses from more than two participants were included and compared, and the fastest

TABLE 1 | Means (with standard deviations [sd] in parentheses) and medians (with median absolute deviation [mad] in parentheses) observed in each condition of Experiment 1 in Meyer et al. (2018), simulated means and medians for pairs of participants, gain (difference between observed and simulated means and medians).

\begin{tabular}{|c|c|c|c|c|c|c|c|}
\hline \multirow[t]{2}{*}{ Display } & \multirow[t]{2}{*}{ Response } & \multicolumn{2}{|c|}{ Observed responses } & \multicolumn{2}{|c|}{ Simulated pairs (winners) } & \multicolumn{2}{|c|}{ Gain } \\
\hline & & Mean (sd) & Median (mad) & Mean (sd) & Median (mad) & Mean & Median \\
\hline Multi-color & Negative & $417 \mathrm{~ms}(177)$ & 325 ms (133) & 325 ms (107) & $276 \mathrm{~ms}(77)$ & $92 \mathrm{~ms}$ & $48 \mathrm{~ms}$ \\
\hline Multi-color & Affirmative & 344 ms (172) & 245 ms (112) & 252 ms (103) & $217 \mathrm{~ms}(71)$ & $92 \mathrm{~ms}$ & $28 \mathrm{~ms}$ \\
\hline Monochrome & Negative & 200 ms (249) & 160 ms (281) & 72 ms (179) & 85 ms (189) & $128 \mathrm{~ms}$ & $75 \mathrm{~ms}$ \\
\hline Monochrome & Affirmative & 234 ms (241) & 200 ms (181) & 96 ms (182) & 134 ms (191) & $149 \mathrm{~ms}$ & $66 \mathrm{~ms}$ \\
\hline
\end{tabular}



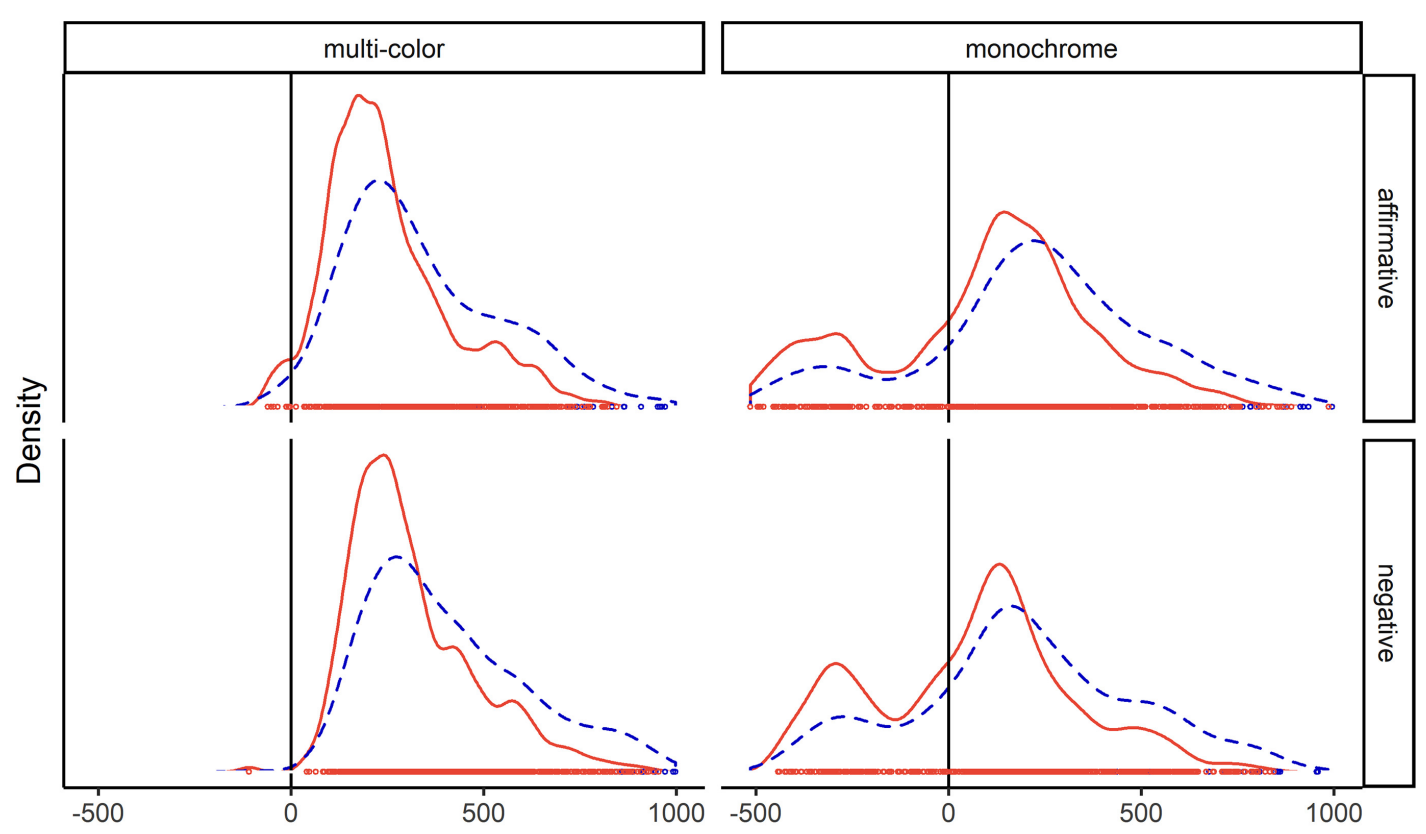

FIGURE 1 | Density plots of all response times (blue) and "winning" times (orange) per condition. Data from Meyer et al. (2018, Experiment 1).

response time was selected. The simulations were run in the same way as described above, but instead of pairs, we generated sets of up to ten participants. Figure 2 shows how the mean of the "winning" response times changed with increasing group size. The mean response times first decreased substantially, but plateaued at a group size of five or six participants because the dataset included only a few valid response times below the plateau. In other words, since the task in, for instance, the multicolor condition could not reliably be completed correctly in less than $200 \mathrm{~ms}$ after the offset of the question, the number of data points entering the comparison did not reduce the mean "winning" response times below that plateau.

The goal of the simulations was to show how the recorded mean response times would change when, instead of including all responses, only the fastest, "winning," response per trial from a sample of two or more participants was recorded. The key point-that the mean of the fastest response time is bound to be shorter than the average time of all response times-could have been made without the simulations. However, we hope that the simulations make the point more apparent for the reader. In addition, we obtained estimates of how much the mean and median response times across all responses versus the fastest responses differed from each other. Whether similar values would be obtained for other datasets remains to be seen. The differences should depend on the properties of the response distributions. When the participants already perform, on average, close to the human performance limit and when there are few slow responses due to, for instance, lapses of attention, the difference will be smaller than when the participants respond more slowly, and/or more erratically. However, regardless of the properties of the distributions, the mean of the fastest response times will always be lower than the mean of all response times.
We simulated the effect of selecting all responses versus a subset of the responses of the participants in an experiment. We did not simulate any changes in the behavior of the participants

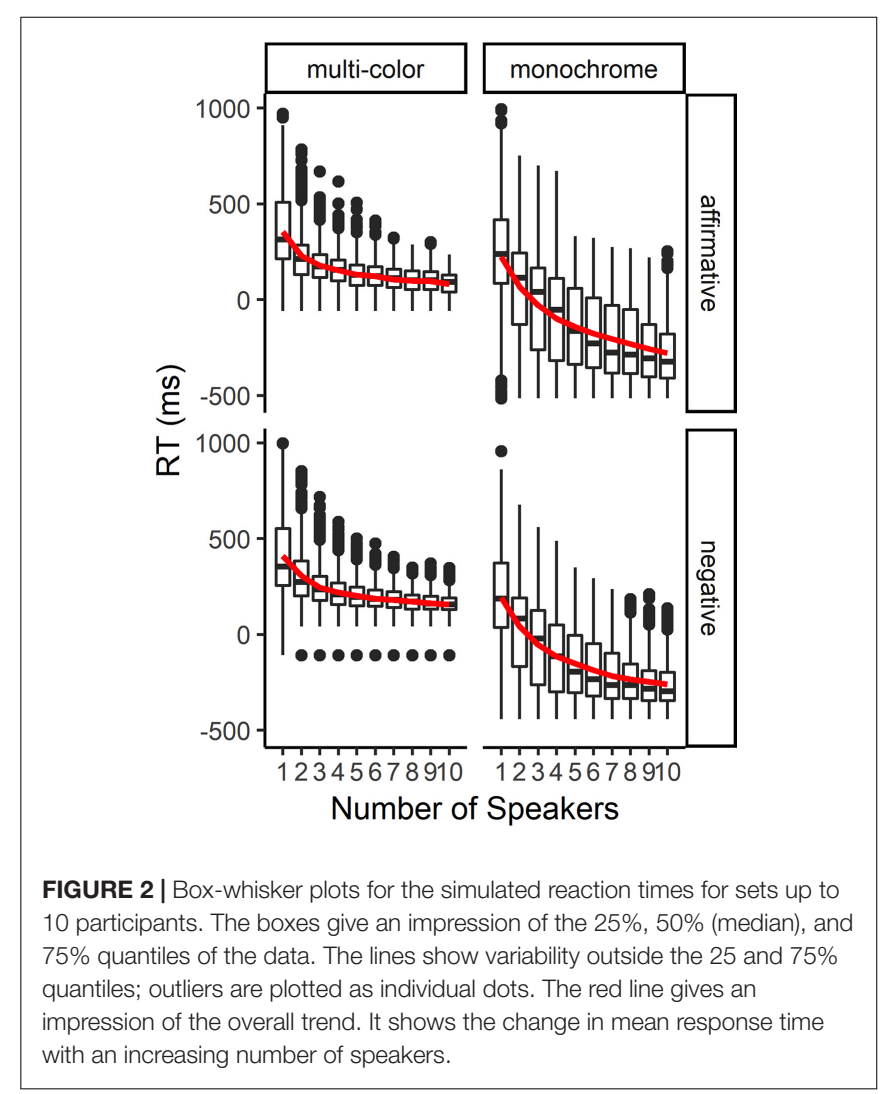


that might occur when they actually compete with each other. Whether and how competition affects participants' response times in a laboratory setting can be assessed in further work. The point of the simulations was to illustrate the potential impact of a difference in the data from laboratory experiments on speech planning and corpora of conversational turn-taking, which often include multi-speaker conversations. The experimental data include all response times, but, as explained before, the corpus data typically only include the fastest responses of speakers competing for the floor. This may contribute to explaining why turn-taking in multi-party conversations appears to be so fast, compared to participants' response times in laboratory experiments.

Note (Figure 2): Outliers deviating more than 2.5 SD from the condition mean were removed from the dataset. For the negativemulti-color condition, the lower limit was $112 \mathrm{~ms}$. One outlier ( $-109 \mathrm{~ms}$, one response from one of the participants) was not filtered out. As this is always the fastest response in any pair/set, this same outlier appears in every box plot.

\section{STUDY 2: CORPUS STUDY}

The aim of the corpus study was to examine how group size may influence turn transition times, i.e., the gaps and overlaps between turns in conversation. We expected turn transitions to be faster in triadic than in dyadic conversations. This should be the case for two reasons: First, in a triadic conversation two speakers may plan a response to a turn but only the fastest response enters the analysis and thus determines the turn transition time. As shown by the above simulations, the mean of these fastest turn transition times should be shorter than the mean of all turn transition times from responses provided by a single speaker in a dyadic conversation. Second, competition for the turn is more likely to arise in triadic than dyadic conversations. These influences should affect turn transition times in non-QR transitions, i.e., turn transitions that are not questions followed by responses. We did not expect a strong group size effect for question-response sequences ( $Q R$ transitions), since here the current speaker often selects a next speaker, thus reducing competition for the floor.

\section{Materials and Methods}

\section{Participants and Corpus Creation}

We analyzed a corpus of 12 dyadic and 12 triadic conversational interactions, each about $20 \mathrm{~min}$ in length. The conversations were selected from a larger corpus, based on the amount of pre-existing relevant annotation from a previous study (Holler and Kendrick, 2015). All conversations involved acquainted native speakers of English, with each participant forming part of one group only (i.e., there were 36 unique speakers in total, two thirds who took part in both the dyadic and the triadic conversation, and one third only in the triadic conversation). The participants' ages ranged from 18 to 68 years (21 female).

Participants arrived in groups of three and were recorded while participating first in a 20 -min triadic conversation followed by a 20 -min dyadic conversation. To create the latter, one of the three people was asked to leave the recording laboratory. Throughout the session, the participants' eye movements were recorded to address questions outside of the scope of this paper (e.g., Holler and Kendrick, 2015; Kendrick and Holler, 2017). Which participant was excluded from the second part of the session depended solely on the quality of the eye movement data acquired in the first session.

The conversations were unscripted and unprompted in terms of topic. Participants were left alone in the recording laboratory and asked to talk to each other as if they were engaging in casual conversation outside of the laboratory environment. They could talk about anything they liked, except for topics which might make their partners feel uncomfortable, for instance for ethical reasons. The entire test session per group lasted around $2 \mathrm{~h}$, including set-up, instructions, two 20-min conversations, and obtaining a range of questionnaire measures at the end of the session, which were not used in the present analyses.

The recordings were made in a laboratory setting at the Max Planck Institute for Psycholinguistics in Nijmegen (Netherlands) and later, when recruitment of native English speakers in Nijmegen became too slow, at the University of Manchester (Manchester, United Kingdom). The audio recordings were made using uni-directional head-mounted microphones (Shure SM10A) which are suitable for detailed phonetic analyses of the audio signals from the individual speakers. For details on the laboratory set-up and equipment see Holler and Kendrick (2015). The two phases of data collection were approved by the Social Sciences Faculty Ethics Committee, Radboud University Nijmegen and the School of Psychological Sciences Ethics Committee, University of Manchester, respectively.

\section{Measurement of Turn Transition Times}

We distinguished turn transition times for question-response sequences (QR transitions) from other turn transitions (non-QR transitions). For the analysis of QR transitions, the full $20 \mathrm{~min}$ of each conversation (i.e., $480 \mathrm{~min}$ in total) were selected. To identify QR transitions we used the coding scheme developed by Stivers and Enfield (2010), which uses both formal criteria (e.g., syntactic marking) and functional criteria (i.e., the utterance seeks to elicit information). This resulted in $459 \mathrm{QR}$ transitions for the dyads (mean $=38.25, \mathrm{SD}=18.85)$ and 497 for the triads (mean $=41.42, \mathrm{SD}=23.63)$.

For the analysis of non-QR transitions, the first 5 min of each conversation were selected (i.e., $120 \mathrm{~min}$ in total). All speaker changes were included, except for the following categories: (a) QR transitions; (b) backchannel responses, such as "mhm," "uhu" (Yngve, 1970; also referred to as continuers by Schegloff, 1982, such utterances are usually considered as passing up the opportunity to take the turn), (c) turn transitions in which the first speaker ended their turn mid-speech due to being interrupted, and (d) turn transitions that involved extended silence, for instance because speakers were thinking of a new topic (e.g., Hoey, 2015); (e) transitions where the coder could not confidently identify first and next speaker because of too much overlapping talk; (f) transitions where the next turn started with 
laughter. This left 290 turn transitions for the dyads (per dyad mean $=24.17, \mathrm{SD}=11.42$ ) and 259 turn transitions for the triads (per triad mean $=21.58, \mathrm{SD}=9.41$ ) for the analyses.

Turn transitions were measured from the offset of vocalization of the first turn to the onset of vocalization of the next turn. This meant that turn transitions involving overlap resulted in negative numbers, and turn transitions involving a gap resulted in positive numbers. Vocalizations were any form of verbal utterance. Audible inbreaths, coughs, and such like were excluded (see also Kendrick and Torreira, 2015). The onsets and offsets of the turns were determined through inspection of their waveforms and spectrograms using Praat (Version 6.1; Boersma and Weenink, 2001). The corpus was annotated manually to select certain types of contributions and excluded others, such as to exclude backchannel responses (see above). These may look like speaker switches, but since they invite the current speaker to continue, they must be distinguished from actual turns. As a result, our analyses are based on a smaller corpus than may be achievable based on automatic coding of speaker contributions, but it allows us to draw clearer conclusions.

\section{Statistical Analysis}

We excluded as outlier turn transition times deviating by more than 1.5 times the interquartile range (i.e., $>$ q0.75 $+1.5 \times$ IQR or $<\mathrm{q} 0.25-1.5 \times \mathrm{IQR})$. This was done separately for the dyads and the triads, combining $\mathrm{QR}$ and non-QR transitions within those groups. There were 90 outliers (5.98\% of the data), 67 QR transitions and 23 non-QR transitions. The data included in the analysis were characterized by a residuals distribution low in skewness and kurtosis (see OSF analysis script) with values falling well within the range of those acceptable for a normal distribution. We thus considered the assumptions underlying linear mixed effects models met. Means, medians, and modes have been calculated to give a comprehensive description of the data (modes were calculated based on Gaussian kernel density estimates, function "locmodes," multimode package).

The data were analyzed using $\mathrm{R}$ (Version 4.0.4; $\mathrm{R}$ Core Team, 2020) and the lme4 package (Version 1.1.27; Bates et al., 2015). A linear mixed effects model was fitted to predict turn transition duration, with group size (coded using sum-to-zero contrasts with dyads coded as +0.5 and triads coded as -0.5) and transition type (also coded using sumto-zero contrast, with QR coded as +0.5 and non-QR coded as -0.5$)$ as fixed effects as well as their interaction. Model diagnostics showed that this model failed to predict negative turn transition durations for most groups, despite the presence of many negative turn transitions in all groups. Therefore, an additional term for "overlap" was added to the model to indicate whether a particular turn transition was positive or negative (see Table 2), resulting in a substantially improved model fit $\left[\chi^{2}(7)=1124.1, p<0.001\right]$. For the participant taking the turn, by-participant intercepts and slopes were included for transition type and overlap. As the model already showed signs of approximate singularity, no interaction between the by-participant slopes were included. The participant identifiers were distinct for dyads and triads. As such, including a by-participant slope for group size was not possible. However, the by-participant contribution was captured by distinct byparticipant intercepts for each conversation. To calculate $p$-values, Satterthwaite's method was used to compute the denominator degrees of freedom with the lmerTest package (Kuznetsova et al., 2017).

A secondary analysis using a logistic mixed effects model (see Table 3) was conducted to see whether the probability of a negative turn transition duration (i.e., overlap) was influenced by transition type or group size. The structure of this model matched the linear mixed model for the transition duration, but overlap was used as the response instead of as a predictor in the fixed and random effects.

\section{Results and Discussion}

We expected turn transitions to be faster in triads than in dyads, especially for non-QR transitions, that is, transitions that are not question-response sequences. Figures 3, 4 show the distributions of the transition times for $Q R$ and nonQR transitions, respectively, including the means and medians. These measures of central tendency, as well as the distributions displayed in the figures, all point toward the same pattern: dyads and triads did not seem to differ much in their turn timing for QR transitions, but triads had faster turn transition times than dyads for non-QR transitions, largely caused by more overlap (i.e., negative turn transition values).

More precisely, for QR transitions, the distributions strongly overlapped (Figure 3). The means for the two groups differed only by $44 \mathrm{~ms}$, and the medians by $9 \mathrm{~ms}$. Thus, turn transitions were not much faster in triads than dyads. The difference between the modes was even greater. The distributions for non-QR transitions also overlapped, but the distribution for triads was shifted leftward relative to the distribution for dyads (Figure 4). This indicates that relatively large negative turn transitions times (i.e., transitions with substantial speaker overlap) were more likely in triads than dyads. The means for triads and dyads differed by $151 \mathrm{~ms}$, the medians by $97 \mathrm{~ms}$, and the comparison of the dyad mode with the lower triad mode by $91 \mathrm{~ms}$, all favoring the triads. The pattern looks different when considering the higher triad mode, indicating that fast responses were not characteristic for all of the triads' non-QR transitions. However, the overall picture that emerges from the measures of central tendency is that group size markedly affected turn transition times for non-QR transitions, but not for $\mathrm{QR}$ transitions, as we expected.

The statistical models, reported in Tables 2, 3 confirmed this impression. The linear mixed effects model (Table 2) showed no significant effects of transition type or group size, but showed some evidence for faster transition times for triadic conversations without overlap $[\beta=54.5, t(58.9)=-1.97, p=0.053]$. That is, in those cases where turn transitions involved a gap, the gaps for triads were on average $55 \mathrm{~ms}$ shorter than those for dyads. Despite the model revealing no difference in terms of overlap duration for the two groups, we applied a second model to test whether group size nevertheless impacts on the probability of overlap. While overall (i.e., collapsing across group size), nonoverlap was more probable than overlap $(\beta=-0.75, z=-9.51$, 
TABLE 2 | Linear mixed model fit by maximum likelihood for the corpus data.

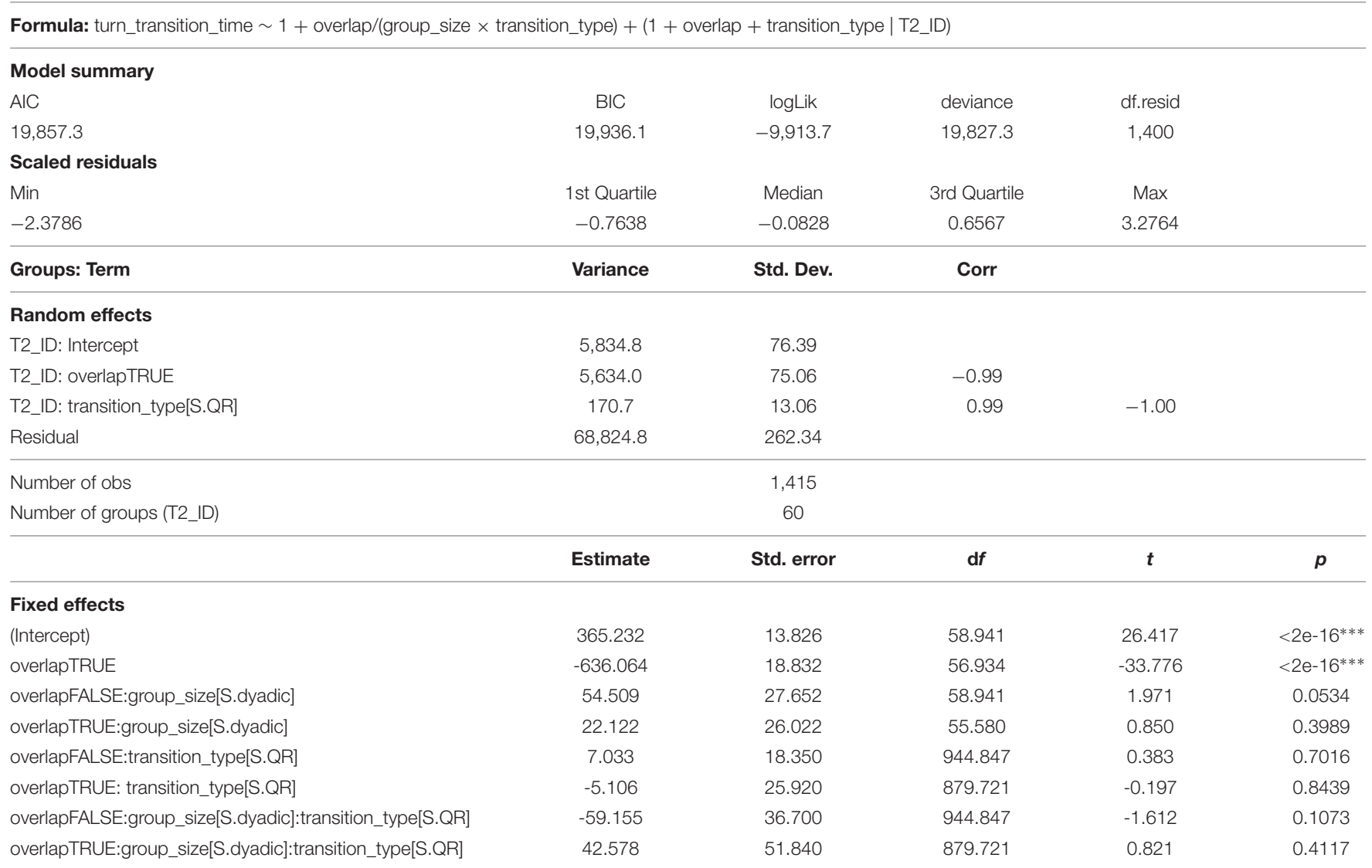



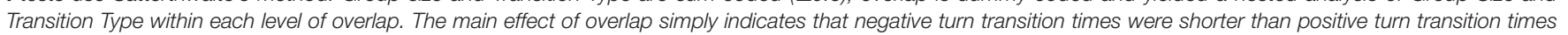
(which is true by definition).

$p<0.001)$, this logistic model highlighted that the difference in mean turn transition duration between the groups was mainly driven by overlap being more probable for triads than dyads $(\beta=-0.39, z=-2.5, p=0.012)$. Additionally, there was an interaction between transition type and group size $(\beta=0.56$, $z=2.16, p=0.03)$. Decomposing this interaction with expected marginal means (using the R package emmeans, Lenth, 2021) showed that overlap was significantly more likely for triads than for dyads in non-QR transitions $(\beta=-0.67, z=3.3$, $p<0.001)$ but not in $\mathrm{QR}$ transitions $(\beta=-0.11, z=-0.56$, $p=0.57)$.

We expected at most a small effect of group size for QR transitions because in such sequences the current speaker often selects the next speaker, which reduces competition for the floor compared to non-QR transitions. Questions in conversation can have many features that single out a particular addressee, such as the use of gaze or the addressee's name (Sacks et al., 1974; Lerner, 1993, 2003). This holds for dyadic and multi-party conversation alike (see Holler and Kendrick, 2015). This means that QR transitions in multi-party conversation are often comparable to QR transitions in dyadic conversation. Bearing this in mind, it is not surprising that we did not observe reliably faster turn transitions for triads than dyads for $\mathrm{QR}$ transitions.
The absence of a group size effect for QR transitions might appear to be inconsistent with the results of the simulations reported above, which yielded strong group size effects for responses to questions. However, the simulations merely illustrated how means of response times change when they are based on all responses versus a subset of responses, specifically the fastest responses per trial from the set of participants. The linguistic properties of the responses used as the basis for the simulations, i.e., that they happened to be responses to questions, played no role for the outcome of the simulations. In other words, the simulations, while based on QR turn transitions, do not reflect the pragmatic attributes associated with such transitions and may, paradoxically, more accurately capture speakers behavior in non-QR transitions.

For non-QR transitions a substantial effect of group size was indeed obtained: The mean turn transition time for dyads was more than double the time observed for triads. The $151 \mathrm{~ms}$ difference corresponds roughly to the duration of a syllable in colloquial English (Greenberg et al., 2003). This difference arose at least in part, from a greater chance of speaker overlap in triads than in dyads. Thus, to the best of our knowledge the present results are the first to show that under comparable conditions, here conversations between acquaintances recorded 
TABLE 3 | Generalized linear mixed model fit by maximum likelihood (Laplace approximation) for the corpus data.

Formula: overlap $\sim 1+$ group size $\times$ transition type $+(1+$ transition type |T2_ID $)$

\begin{tabular}{|c|c|c|c|c|}
\hline \multicolumn{5}{|l|}{ Model summary } \\
\hline $\mathrm{AIC}$ & $\mathrm{BIC}$ & logLik & Deviance & df.resid \\
\hline $1,782.7$ & $1,819.5$ & -884.4 & $1,768.7$ & 1,408 \\
\hline \multicolumn{5}{|l|}{ Scaled residuals } \\
\hline Min & 1st Quartile & Median & 3rd Quartile & Max \\
\hline-1.0534 & -0.7232 & -0.5812 & 1.2107 & 1.9376 \\
\hline Groups: Term & Variance & Std. Dev. & Corr & \\
\hline T2_ID: Intercept & 0.12422 & 0.3525 & & \\
\hline T2_ID: transition_type [QR] & 0.07033 & 0.2652 & -1.00 & \\
\hline Number of obs & & 1415 & & \\
\hline Number of groups (T2_ID) & & 60 & & \\
\hline group_size [S.dyadic] & -0.39172 & 0.15649 & -2.503 & $0.0123^{\star}$ \\
\hline transition_type [S.QR] & 0.13720 & 0.12931 & 1.061 & 0.2887 \\
\hline group size [S.dyadic]:transition_type [S.QR] & 0.55533 & 0.25734 & 2.158 & $0.0309^{*}$ \\
\hline
\end{tabular}

The response family was binomial with a logit link. Group Size and Transition Type are sum coded ( \pm 0.5$)$.

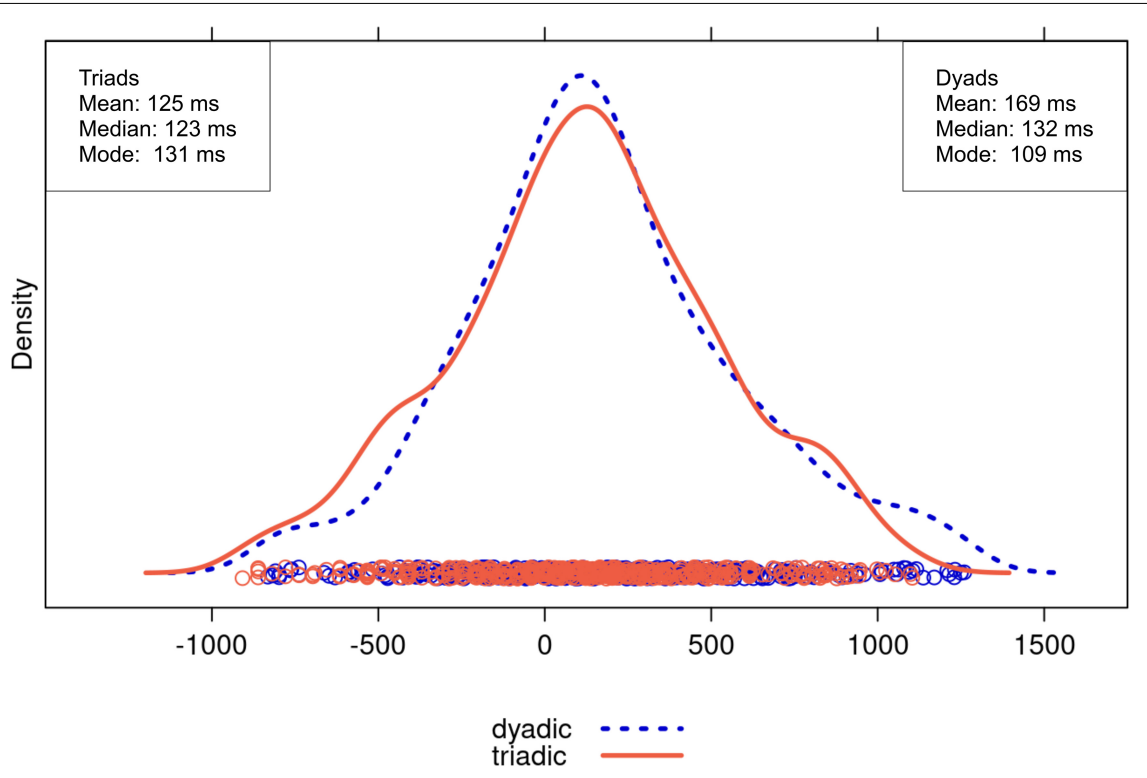

FIGURE 3 | Distribution of turn transition times (in ms) for QR transitions for dyadic (dotted blue) and triadic (orange) conversations (outliers excluded).

in the laboratory, triads, on average, have shorter non-QR turn transitions than dyads.

Of course, this initial finding must be supported by further work. A strength of the current corpus is that the same participants were involved in the triadic and dyadic conversations. An obvious shortcoming is that for all speakers the triadic conversations preceded the dyadic ones. It is conceivable that the observed group size effect for non-QR transitions resulted from fatigue or decreasing motivation to talk in the second session of the study (i.e., the dyads). However, we think this is unlikely because fatigue or decreasing motivation should not selectively affect the non-QR transitions, but also the QR transitions, for which no group size effect was found. Nonetheless, further studies with counterbalanced order of group sizes, or between-participant designs, are needed to separate group size and order effects more conclusively. Moreover, it 


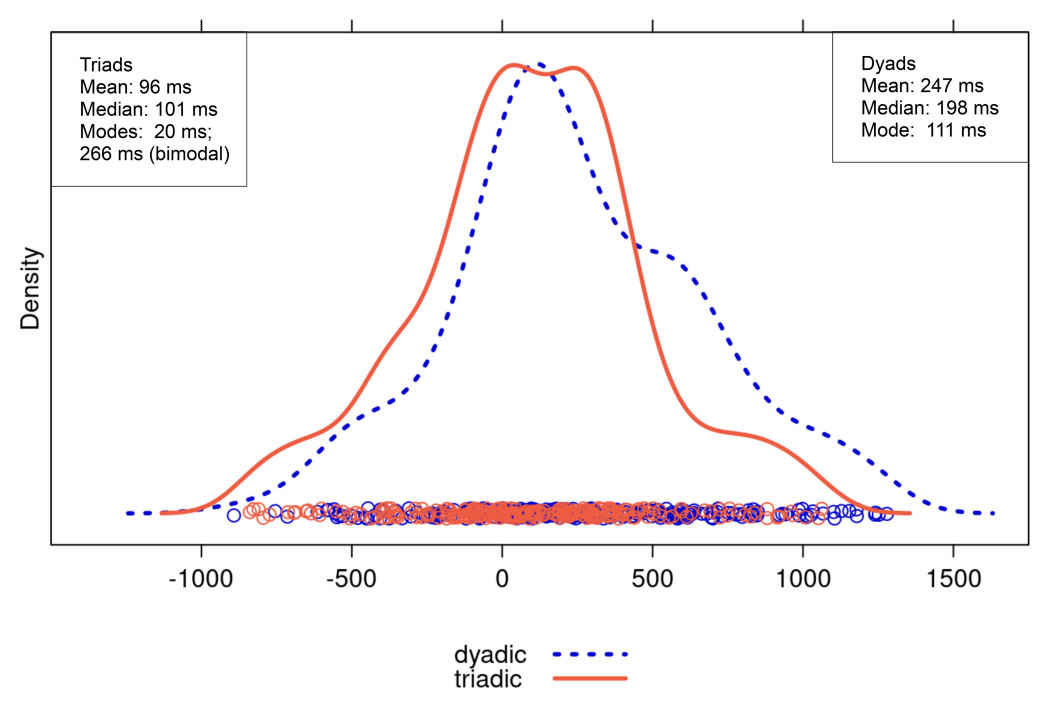

FIGURE 4 | Distribution of turn transition times (in ms) in non-QR transitions for dyadic (dotted blue) and triadic (orange) conversations (outliers excluded).

would also be good to consider larger corpora and corpora of different languages to see how generalizable the present effects are.

\section{GENERAL DISCUSSION}

We set out to investigate how the number of potential next speakers in a conversation may influence turn transition times. The starting point was the observation that in quantitative corpus analyses of multi-party conversation, only the fastest response to a turn enters the analysis. The reason is that participants who needed more time to respond may end up not producing a response, or they may produce it after someone else has taken the next turn, thus precluding it from the analysis. This may partly explain why turn transition times in natural conversations are so short compared to the response times in laboratorybased experimental studies of turn-taking. In the lab, participants are tested individually and all response times (except for some extremely long or short response times), feed into the mean. Indeed, the results of our simulation study confirmed that, up to a set size of about five participants, the more participants were entered into the model as potential responders, the shorter the mean "winning" response time became. This principle-that the mean of a subset of short response times will be lower than the mean of all response times in a dataset-is bound to play a role in multi-party conversation as well.

However, turn transition times may also be shorter in triads than dyads for other reasons, in particular differences in competition for the turn. At those points in conversation where turn transition becomes relevant (i.e., points of semantic, syntactic and pragmatic completion, Sacks et al., 1974; Ford et al., 1996), a current speaker either selects the next speaker or provides other participants with the opportunity to self-select. Since in multi-party conversation the "first starter acquires rights to a turn" (Sacks et al., 1974, p. 704), responding fast pays off. This does not apply to the results of the simulations where competitive behavior does not come into play. Thus, the strong group size effect, of $151 \mathrm{~ms}$ (for the means) seen for non-QR transitions was most likely due to a combination of sampling the fastest responses in the triadic corpus and increased competition for the floor.

The earlier corpus-based literature on turn transition times has concerned dyads, multi-party conversation, or a mixture of the two (e.g., Stivers et al., 2009; de Vos et al., 2015; Girard-Groeber, 2015; Holler et al., 2018). This complicates the comparison of turn transition times to response times obtained in laboratory settings from individually tested participants. We should note, however, that the inclusion of multi-party conversations in some of the corpora does not fully explain why the turn transition times are so much shorter than the response latencies in the laboratory. This is evident from the observation that turn transition times in dyadic conversation are also often shorter than response times of individual participants in the lab (e.g., Knudsen et al., 2020). Thus, there must be other factors at play that facilitate swift responding in natural conversations and/or hinder it in experimental settings. These factors could, for instance, pertain to the speakers' motivation and engagement or to the linguistic structure and predictability of their utterances, as well as to their use of gaze, which has long been claimed to play an important role in turn-taking (Kendon, 1967).

While we have here focused on variables that may contribute to responses in conversation being faster than in the laboratory, there are also variables that occasion slow responses in conversation. These may relate to uncertainty about the content of the response (e.g., Fox and Thompson, 2010), or they may relate to pragmatics. For instance, dispreferred responses are often marked by longer turn transition times than preferred responses (Schegloff, 2007; Kendrick and Torreira, 2015) and initiations of repair (clarification requests resulting from trouble in understanding) generally occur after significant delays (Kendrick, 2015). 
In sum, we propose that the absence of slow responses from typical corpus data, enhanced by the competition for turns, may partly explain the short turn transition times in everyday multiparty conversation. Further work is needed to substantiate this suggestion. In addition, the impact of other variables that may also influence turn timing (e.g., Roberts et al., 2015) remains to be identified. We think this would best be done by combining corpus analyses and experimental work, with the former providing fine-grained descriptive evidence about conversation in different settings and the latter uncovering the cognitive tools at the speakers' disposition.

\section{DATA AVAILABILITY STATEMENT}

The datasets presented in this study can be found in an online repository: https://osf.io/um7ta/?view_only= 9cd734fa2e9346e3aa89b3f958b46ef6.

\section{ETHICS STATEMENT}

The studies involving human participants were reviewed and approved by Social Sciences Faculty Ethics Committee, Radboud

\section{REFERENCES}

Barthel, M., Meyer, A. S., and Levinson, S. C. (2017). Next Speakers Plan Their Turn Early and Speak after Turn-Final “Go-Signals.”. Front. Psychol. 8:393. doi: 10.3389/fpsyg.2017.00393

Barthel, M., Sauppe, S., Levinson, S. C., and Meyer, A. S. (2016). The Timing of Utterance Planning in Task-Oriented Dialogue: evidence from a Novel ListCompletion Paradigm. Front. Psychol. 7:1858. doi: 10.3389/fpsyg.2016.01858

Bates, D., Mächler, M., Bolker, B., and Walker, S. (2015). Fitting Linear MixedEffects Models Using lme4. J. Stat. Softw. 67, 1-48. doi: 10.18637/jss.v067. i01

Beattie, G. W. (1982). Turn-taking and interruption in political interviews: margaret Thatcher and Jim Callaghan compared and contrasted. Semiotica 39, 93-114.

Boersma, P., and Weenink, D. (2001). PRAAT, a system for doing phonetics by computer. Glot Int. 5, 341-345.

Bögels, S., Casillas, M., and Levinson, S. C. (2018). Planning versus comprehension in turn-taking: fast responders show reduced anticipatory processing of the question. Neuropsychologia 109, 295-310. doi: 10.1016/j.neuropsychologia. 2017.12.028

Bögels, S., Magyari, L., and Levinson, S. C. (2015). Neural signatures of response planning occur midway through an incoming question in conversation. Sci. Rep. 5:12881. doi: 10.1038/srep12881

Boiteau, T. W., Malone, P. S., Peters, S. A., and Almor, A. (2014). Interference between conversation and a concurrent visuomotor task. J. Exp. Psychol. Gen. 143, 295-311. doi: 10.1037/a0031858

Branigan, H. P., and Pickering, M. J. (2017). Structural priming and the representation of language. Behav. Brain Sci. 40:e313. doi: 10.1017/ S0140525X17001212

Branigan, H. P., Pickering, M. J., and Cleland, A. A. (2000). Syntactic co-ordination in dialogue. Cognition 75, B13-B25. doi: 10.1016/S0010-0277(99)00081-5

Brennan, S. E., and Clark, H. H. (1996). Conceptual pacts and lexical choice in conversation. J. Exp. Psychol. Learn. Mem. Cogn. 22, 1482-1493. doi: 10.1037/ 0278-7393.22.6.1482

Brown-Schmidt, S., Yoon, S. O., and Ryskin, R. A. (2015). "Chapter Three - People as Contexts in Conversation," in Psychology of Learning and Motivation, ed. B. H. Ross (Cambridge: Academic Press), 59-99.

Clark, H. H. (1996). Using language. New York: Cambridge University Press.
University Nijmegen. The patients/participants provided their written informed consent to participate in this study.

\section{AUTHOR CONTRIBUTIONS}

$\mathrm{JH}$ and AM conceptualized and wrote the manuscript. PA designed the simulation. CD contributed to the design and data collection analyzed in Study 1. KK and JH collected the data for Study 2. KK and MG annotated the data for Study 2. PA analyzed the data for Studies 1 and 2. CD and MG contributed to the analysis for Studies 1 and 2, respectively. All authors contributed to the final version of the manuscript.

\section{ACKNOWLEDGMENTS}

We thank Samantha Rowbotham for help with recruitment for collecting the corpus data at the University of Manchester, Manchester, United Kingdom. We would also like to thank the European Research Council (grants \#773079, JH and \#269484, SCL) for financial support.

Cleland, A. A., Tamminen, J., Quinlan, P. T., and Gaskell, M. G. (2012). Spoken word processing creates a lexical bottleneck. Lang. Cogn. Process. 27, 572-593. doi: 10.1080/01690965.2011.564942

R Core Team (2020). R: A Language and Environment for Statistical Computing. Vienna: R Foundation for Statistical Computing.

Corps, R. E., Crossley, A., Gambi, C., and Pickering, M. J. (2018). Early preparation during turn-taking: listeners use content predictions to determine what to say but not when to say it. Cognition 175, 77-95. doi: 10.1016/j.cognition.2018.01. 015

Corps, R. E., Pickering, M. J., and Gambi, C. (2019). Predicting turn-ends in discourse context. Lang. Cogn. Neurosci. 34, 615-627. doi: 10.1080/23273798. 2018.1552008

de Vos, C., Torreira, F., and Levinson, S. C. (2015). Turn-timing in signed conversations: coordinating stroke-to-stroke turn boundaries. Front. Psychol. 6:268. doi: 10.3389/fpsyg.2015.00268

Ferguson, N. (1977). Simultaneous speech, interruptions and dominance. Br. J. Soc. Clin. Psychol. 16, 295-302.

Ferreira, V. S., and Pashler, H. (2002). Central bottleneck influences on the processing stages of word production. J. Exp. Psychol. Learn. Mem. Cogn. 28, 1187-1199. doi: 10.1037/0278-7393.28.6.1187

Ford, C. E., Fox, B. A., and Thompson, S. A. (1996). Practices in the construction of turns: the "TCU” revisited. Pragmatics 6, 427-454. doi: 10.1075/prag.6.3.07for

Fox, B. A., and Thompson, S. A. (2010). Responses to Wh-Questions in English Conversation. Res. Lang. Soc. Interact. 43, 133-156. doi: 10.1080/ 08351811003751680

French, P., and Local, J. (1983). Turn-competitive incomings. J. Pragmat. 7, 17-38. doi: 10.1016/0378-2166(83)90147-9

Garrod, S., and Anderson, A. (1987). Saying what you mean in dialogue: a study in conceptual and semantic co-ordination. Cognition 27, 181-218. doi: 10.1016/ 0010-0277(87)90018-7

Garrod, S., and Pickering, M. J. (2009). Joint Action, Interactive Alignment, and Dialog. Top. Cogn. Sci. 1, 292-304. doi: 10.1111/j.1756-8765.2009.01020.x

Girard-Groeber, S. (2015). The management of turn transition in signed interaction through the lens of overlaps. Front. Psychol. 6:741. doi: 10.3389/fpsyg.2015. 00741

Greenberg, S., Carvey, H., Hitchcock, L., and Chang, S. (2003). Temporal properties of spontaneous speech-a syllable-centric perspective. J. Phon. 31, 465-485. doi: 10.1016/j.wocn.2003.09.005 
Griffin, Z., and Bock, K. (2000). What the Eyes Say About Speaking. Psychol. Sci. 11, 274-279. doi: 10.1111/1467-9280.00255

Healey, P., Purver, M., and Howes, C. (2014). Divergence in Dialogue. PLoS One 9:e98598. doi: 10.1371/journal.pone.0098598

Heldner, M., and Edlund, J. (2010). Pauses, gaps and overlaps in conversations. J. Phon. 38, 555-568. doi: 10.1016/j.wocn.2010.08.002

Hoey, E. (2015). Lapses: how People Arrive at, and Deal With, Discontinuities in Talk. Res. Lang. Soc. Interact. 48, 430-453. doi: 10.1080/08351813.2015.1090116

Holler, J., and Kendrick, K. H. (2015). Unaddressed participants' gaze in multiperson interaction: optimizing recipiency. Front. Psychol. 6:98. doi: 10.3389/ fpsyg.2015.00098

Holler, J., Kendrick, K. H., and Levinson, S. C. (2018). Processing language in faceto-face conversation: questions with gestures get faster responses. Psychon. Bull. Rev. 25, 1900-1908. doi: 10.3758/s13423-017-1363-z

Indefrey, P., and Levelt, W. (2004). Indefrey, P. \& Levelt, W.J.M. The spatial and temporal signatures of word production components. Cognition 92, 101-144. doi: 10.1016/j.cognition.2002.06.001

Kendon, A. (1967). Some functions of gaze-direction in social interaction. Acta Psychol. 26, 22-63. doi: 10.1016/0001-6918(67)90005-4

Kendrick, K. H. (2015). The intersection of turn-taking and repair: the timing of other-initiations of repair in conversation. Front. Psychol. 6:250. doi: 10.3389/ fpsyg.2015.00250

Kendrick, K. H., and Holler, J. (2017). Gaze direction signals response preference in conversation. Res. Lang. Soc. Interact. 50, 12-32. doi: 10.1080/08351813.2017. 1262120

Kendrick, K. H., and Torreira, F. (2015). The Timing and Construction of Preference: a Quantitative Study. Discourse Process. 52, 255-289. doi: 10.1080/ 0163853X.2014.955997

Knudsen, B., Creemers, A., and Meyer, A. S. (2020). Forgotten Little Words: how Backchannels and Particles May Facilitate Speech Planning in Conversation?. Front. Psychol. 11:3071. doi: 10.3389/fpsyg.2020.593671

Konopka, A. E. (2012). Planning ahead: how recent experience with structures and words changes the scope of linguistic planning. J. Mem. Lang. 66, 143-162. doi: 10.1016/j.jml.2011.08.003

Kurtić, E., Brown, G. J., and Wells, B. (2013). Resources for turn competition in overlapping talk. Speech Commun. 55, 721-743. doi: 10.1016/j.specom.2012.10. 002

Kuznetsova, A., Brockhoff, P. B., and Christensen, R. H. B. (2017). lmerTest Package: tests in Linear Mixed Effects Models. J. Stat. Softw. 82, 1-26. doi: 10.18637/jss.v082.i13

Lenth, R. V. (2021). emmeans: Estimated Marginal Means, aka Least-Squares Means. $R$ package Version 1.6.1. Available online at: https://CRAN.R-project. org/package $=$ emmeans

Lerner, G. H. (1993). Collectivities in action: establishing the relevance of conjoined participation in conversation. Text 13, 213-246. doi: 10.1515/text.1.1993.13.2. 213

Lerner, G. H. (2003). Selecting next speaker: the context-sensitive operation of a context-free organization. Lang. Soc. 32, 177-201. doi: 10.1017/ S004740450332202X

Levinson, S. C. (2016). Turn-taking in Human Communication - Origins and Implications for Language Processing. Trends Cogn. Sci. 20, 6-14. doi: 10.1016/ j.tics.2015.10.010

Levinson, S. C., and Torreira, F. (2015). Timing in turn-taking and its implications for processing models of language. Front. Psychol. 6:731. doi: 10.3389/fpsyg. 2015.00731

Meyer, A. S., Alday, P. M., Decuyper, C., and Knudsen, B. (2018). Working Together: contributions of Corpus Analyses and Experimental Psycholinguistics to Understanding Conversation. Front. Psychol. 9:525. doi: $10.3389 /$ fpsyg. 2018.00525
Pickering, M. J., and Garrod, S. (2004). Toward a mechanistic psychology of dialogue. Behav. Brain Sci. 27, 169-190.

Roberts, S. G., Torreira, F., and Levinson, S. C. (2015). The effects of processing and sequence organization on the timing of turn taking: a corpus study. Front. Psychol. 6:509. doi: 10.3389/fpsyg.2015.00509

Sacks, H., Schegloff, E. A., and Jefferson, G. (1974). A Simplest Systematics for the Organization of Turn-Taking for Conversation. Language 50:696. doi: 10.2307/ 412243

Schegloff, E. (1982). "Discourse as an interactional achievement: Some uses of 'uh huh' and other things that come between sentences," in Analyzing discourse: Text and talk, ed. D. Tannen (Washington: Georgetown University Press)), 71-93.

Schegloff, E. A. (2000). Overlapping talk and the organization of turntaking for conversation. Lang. Soc. 29, 1-63. doi: 10.1017/S004740450000 1019

Schegloff, E. A. (2001). "Accounts of conduct in interaction: Interruption, overlap, and turn-taking," in Handbook of Sociological Theory, ed. J. H. Turner (Boston: Springer), $287-321$

Schegloff, E. A. (2007). Sequence Organization in Interaction: A Primer in Conversation Analysis. Cambridge: Cambridge University Press.

Sjerps, M. J., and Meyer, A. S. (2015). Variation in dual-task performance reveals late initiation of speech planning in turn-taking. Cognition 136, 304-324. doi: 10.1016/j.cognition.2014.10.008

Stivers, T., and Enfield, N. J. (2010). A coding scheme for question-response sequences in conversation. J. Pragmat. 42, 2620-2626. doi: 10.1016/J.PRAGMA. 2010.04.002

Stivers, T., Enfield, N. J., Brown, P., Englert, C., Hayashi, M., Heinemann, T., et al. (2009). Universals and cultural variation in turn-taking in conversation. Proc. Natl. Acad. Sci. U. S. A. 106:10587. doi: 10.1073/pnas.090361 6106

ten Bosch, L., Oostdijk, N., and de Ruiter, J. P. (2004). "Durational Aspects of TurnTaking in Spontaneous Face-to-Face and Telephone Dialogues," in Text, Speech and Dialogue, eds P. Sojka, I. Kopeèek, and K. Pala (Berlin: Springer Berlin Heidelberg), 563-570.

Trimboli, C., and Walker, M. B. (1984). Switching pauses in cooperative and competitive conversations. J. Exp. Soc. Psychol. 20, 297-311. doi: 10.1016/00221031(84)90027-1

Yngve, V. (1970). "On getting a word in edgewise," in Papers from the Sixth Regional Meeting [of the] Chicago Linguistic Society, ed. R. I. Binnick (Chicago: University of Chicago Department of Linguistics), 568.

Conflict of Interest: The authors declare that the research was conducted in the absence of any commercial or financial relationships that could be construed as a potential conflict of interest.

Publisher's Note: All claims expressed in this article are solely those of the authors and do not necessarily represent those of their affiliated organizations, or those of the publisher, the editors and the reviewers. Any product that may be evaluated in this article, or claim that may be made by its manufacturer, is not guaranteed or endorsed by the publisher.

Copyright (c) 2021 Holler, Alday, Decuyper, Geiger, Kendrick and Meyer. This is an open-access article distributed under the terms of the Creative Commons Attribution License (CC BY). The use, distribution or reproduction in other forums is permitted, provided the original author(s) and the copyright owner(s) are credited and that the original publication in this journal is cited, in accordance with accepted academic practice. No use, distribution or reproduction is permitted which does not comply with these terms. 